Pacific Journal of Mathematics

HYPOTOPOLOGICAL SPACES AND THEIR EMBEDDINGS IN
LATTICES WITH BIRKHOFF INTERVAL TOPOLOGY 


\title{
HYPOTOPOLOGICAL SPACES AND THEIR EMBEDDINGS IN LATTICES WITH BIRKHOFF INTERVAL TOPOLOGY
}

\author{
KIRBY A. BAKER
}

\begin{abstract}
Important classes of topological spaces have topologies which are induced by a generating collection of closed subsets; typical examples are $k$-spaces, sequential spaces with unique sequential limits, and lattices with the Birkhoff interval topology. This paper proceeds by axiomatizing this construction - a set with a specified generating collection of closed subsets is called a "hypotopological space." The Birkhoff interval topology is then studied in these terms. A natural embedding of hypotopological spaces in conditionally complete, atomic, distributive lattices with Birkhoff interval topology is derived. This embedding is used to show that lattices with Birkhoff interval topology have the same nontrivial subspace and product properties as $k$-spaces and sequential spaces. In particular, we answer in the negative a question first raised by Birkhoff, namely, whether the Birkhoff interval topology is preserved under the formation of the product of two lattices.
\end{abstract}

The Birkhoff interval topology was defined in [5]. It is one of the most natural of the various topologies which have been proposed for lattices, and yet is one of the least amenable to explicit calculation. Certain cases have proven tractable: For partially ordered sets with universal bounds (0 and 1), the Birkhoff interval topology coincides with the Frink interval topology [15], under which the closed intervals form a subbase for the closed sets. In general, however, it is difficult to determine the closure of a given subset of a lattice with respect to this topology. It is for this reason that several basic questions regarding subspaces and products have proven elusive for the Birkhoff interval topology.

Subspace and product properties of $k$-spaces and sequential spaces have been studied by Franklin [12, 13], Cohen [9], Dowker [10], Dudley [11], Michael $[19,20]$, and others. Birkhoff noted that a conditionally complete lattice is itself a $k$-space under his interval topology [5, Th. 3]. For the Birkhoff interval topology, products of chains were studied by Alo and Frink [1]. In particular, they answered Birkhoff's question in the infinite case by showing that the Birkhoff interval topology is not preserved under infinite products of chains. The example derived in the present paper answers Birkhoff's question for the finite case (see Corollary 6.8 below). 


\section{Hypotopological spaces.}

Definition 2.1. A hypotopological space $X$ is a set $X$ together with a specified collection $\mathscr{K}(X)$ of subsets of $X$ satisfying

(A1) $\varnothing \in \mathscr{K}(X)$,

(A2) if $C_{1}, C_{2} \in \mathscr{K}(X)$ then $C_{1} \cup C_{2} \in \mathscr{K}(X)$,

(A3) if $\left\{C_{\alpha}\right\}$ is a nonempty family of elements of $\mathscr{K}(X)$, then $\cap_{\alpha} C_{\alpha} \in \mathscr{K}(X)$

(A4) $\mathscr{K}(X)$ is a covering of $X$.

We shall call $\mathscr{K}(X)$ a hypotopology on $X$. The terminology is motivated by the observation that $\mathscr{K}(X)$ would be a (closed set) topology on $X$ in the presence of the additional condition $X \in \mathscr{K}(X)$.

If $X$ is a hypotopological space, let $\mathscr{K}(X): \mathscr{K}(X)$ denote $\{V \cong$ $X: V \cap C \in \mathscr{K}(X)$ for all $C \in \mathscr{K}(X)\}$. (The notation is that of residuation in lattices [5, p. 327].) Then $\mathscr{K}(X): \mathscr{K}(X)$ is a (closed set) topology on $X$, called the topology induced by the hypotopology $\mathscr{K}(X)$. Since for each $C \in \mathscr{K}(X)$ the intersections with $C$ of elements of $\mathscr{K}(X)$ form a (closed set) topology on $C$, the induced topology on $X$ is an instance of a topology generated by a collection of subspaces ([18, App. D), [8, p. 615], [7, p. 33]); it is the strongest topology on $X$ which agrees with the topology of each $C \in \mathscr{K}(X)$. (Whitehead, in a different terminology, would call such a topology "weak" [21, §5].)

Following Birkhoff [5], we shall refer to the elements of $\mathscr{K}(X)$ : $\mathscr{K}(X)$ as closed subsets of $X$ and to any subset of an element of $\mathscr{K}(X)$ as a bounded subset of $X$. The closed bounded subsets of $X$ are then precisely the elements of $\mathscr{K}(X)$. Complements of closed sets will be called "open," as usual.

If $X$ is a hypotopological space, the induced topology on $X$ can be obtained by representing $X$ as a quotient [7, p. 40] of the disjoint union $X^{\prime}$ of the sets $C \in \mathscr{C}(X)$, each regarded as a topological space. Here the quotient map $\mu_{X}: X^{\prime} \rightarrow X$ is the unique map which restricts to the identity on each $C \in \mathscr{K}(X)$. Following [4], we shall call $\mu_{X}$ the canonical presentation of $X$ (under the induced topology) as a quotient.

REMARK. The bounded subsets of $X$ form a "boundedness [system]" in the sense of $\mathrm{Hu}[16, \mathrm{p} .184]$. Conversely, if a boundedness on a topological space $X$ is such that one-point subsets are bounded and closures of bounded sets are bounded, then the closed bounded sets constitute a hypotopology on $X$ with the same definition of "bounded".

EXAMPLE 1. Let $\omega+1$ be the second infinite ordinal, with the order topology. If $X$ is a topological space, then intersections of finite 
unions of subspaces of $X$ homeomorphic to $\omega+1$ form a hypotopology on $X$, the sequential hypotopology. $X$ is a sequential topological space with unique sequential limits if and only if the induced topology is the original topology on $X$ [12, Proposition 1.1, p. 108].

EXAmple 2. If $X$ is a topological space, then the closed compact subsets of $X$ form a hypotopology on $X$ which we shall call the full $k$-hypotopology. $X$ is a $k$-space if and only if the induced topology is the original topology on $X[17, \mathrm{p} .230]$. If $X$ is a complete uniform space which is a $k$-space (e.g., if $X$ is a complete metric space), then the bounded subsets of $X$ are those which are totally bounded in the sense of uniform spaces $[17, \mathrm{p} .198]$.

More generally, if $X$ is any hypotopological space for which all $C \in \mathscr{K}(X)$ are compact, then $X$ is a $k$-space under the induced topology, and we shall call $\mathscr{K}(X)$ a k-hypotopology on $X$.

Example 3. If $X$ is a partially ordered set, let $\mathscr{K}(X)$ be the hypotopology on $X$ consisting of all intersections of finite unions of intervals $[a, b]=\{x \in X: a \leqq x \leqq b\} . \mathscr{K}(X)$ will be called the interval hypotopology on $X$. The induced topology is the Birkhoff interval topology on $X$ [5]. If $X$ is bidirected (i.e., if $X$ and its dual are directed), then the bounded subsets of $X$ are those which are bounded above and below in the sense of partial order.

Finally, any topological space $X$ can be regarded as a hypotopological space, with $\mathscr{K}(X)$ being the collection of closed subsets of $X$.

Most elementary definitions and constructions for topological spaces, when phrased in terms of closed sets, have natural generalizations to hypotopological spaces:

If $X$ is a hypotopological space, a collection $\mathscr{B}$ of subsets of $X$ is subbase for $\mathscr{K}(X)$ if $\mathscr{K}(X)$ is the smallest hypotopology on $X$ which contains $\mathscr{B} ; \mathscr{K}(X)$ can be obtained as the collection of arbitrary intersections of finite unions of elements of $\mathscr{B}$. (Here and elsewhere the phrase "arbitrary intersection" should be interpreted to exclude the intersection of no sets.) Thus intervals $[a, b]$ are a subbase for the interval hypotopology.

If $S \subseteq X$, the relative hypotopology $\mathscr{C}(S)$ on $S$ is given by

$$
\mathscr{K}(S)=\{S \cap C: C \in \mathscr{K}(X)\} .
$$

If $S$ is bounded, $\mathscr{K}(S)$ is thus a (closed-set) topology on $S$. If $\left\{X_{\alpha}\right.$, $\alpha \in A\}$ is a family of hypotopological spaces, their disjoint sum $\sum_{\alpha} X_{\alpha}$ has the hypotopology with subbase $\bigcup_{\alpha} \mathscr{C}\left(X_{\alpha}\right)$ (where the $X_{\alpha}$ are regarded as disjoint). The direct product $\Pi_{\alpha} X_{\alpha}$ has the hypotopology determined by the subbase consisting of "boxes" $\Pi_{\alpha} C_{\alpha}$, where $C_{\alpha} \in \mathscr{K}\left(X_{\alpha}\right)$ 
for all $\alpha$.

Homeomorphisms and embeddings of hypotopological spaces are defined in the obvious way. More generally, a morphism $f: X \rightarrow Y$ of hypotopological spaces is a function $f$ which is continuous with respect to the induced topologies on $X$ and $Y$ and which carries bounded subsets of $X$ into bounded subsets of $Y$. Hypotopological spaces and their morphisms form a category in which direct products and disjoint sums, as defined above, do correspond to direct products and direct sums defined categorically $[14, p .76]$.

We shall say that a hypotopological space $X$ is Hausdorff if the induced topology is Hausdorff; a $T_{1}$ hypotopological space is similarly defined.

3. Subspaces of hypotopological spaces. It is well known that subspaces of sequential topological spaces need not be sequential [12, Example 1.8] and that subspaces of $k$-spaces need not be $k$-spaces [17, p. 240]. For hypotopological spaces in general the situation can be analyzed as follows.

Let $X$ be a hypotopological space, and let $S$ be a subspace with the relative hypotopology $\mathscr{K}(S)$. Then there arise two conceivably different topologies on $S$.

(1) the topology $\mathscr{K}(S): \mathscr{K}(S)$ induced by $\mathscr{K}(S)$;

(2) the relativization to $S$ of the induced topology $\mathscr{K}(X): \mathscr{K}(X)$ on $X$. Topology (1) is always at least as strong as topology (2).

DEFINITION 3.1. If topologies (1) and (2) are identical, we shall say that $S$ is a strong subspace of $X$.

The following fact is immediate from the definition of the quotient $\operatorname{map} \mu_{X}$.

Lemma 3.2. Let $\mu_{X}: X^{\prime} \rightarrow X$ be the canonical presentation of $X$ (under its induced topology) as the quotient of a topological sum, and let $S \subseteq X$. Then $S$ is strong if and only if $\mu_{X}$ restricted to $\mu_{X}^{-1}(S)$ is a quotient map.

By means of this lemma, the following facts are readily verified.

THEOREM 3.3. Let $X$ be a hypotopological space. Then

(i) every closed subset of $X$ is strong;

(ii) every open subset of $X$ is strong;

(iii) every bounded subset of $X$ is strong.

THEOREM 3.4. Let $X$ be a hypotopological space, let $Z \cong Y \subseteq X$, 
and let $Z$ and $Y$ have the relative hypotopologies. Then in $Y$;

(i) (relative heredity) if $Z$ is strong in $X$, then $Z$ is strong

(ii) (transitivity) if $Z$ is strong in $Y$ and $Y$ is strong in $X$, then $Z$ is strong in $X$.

REMARK. If a subset $S$ of a $k$-space is itself a $k$-space, then it is strong, but not conversely - a relatively closed bounded set may no longer be compact. However, a subspace of a sequential space is strong if and only if it is sequential. Theorem 3.3 therefore generalizes Proposition 1.9 of [12], to the effect that closed subsets and open subsets of sequential spaces are sequential.

In a number of important examples, the following theorems show that all subsets are strong. A map $f: Y \rightarrow Z$ of topological spaces is pseudo-open if $f(U)$ is a neighborhood of $p \in Z$ whenever $U$ is a neighborhood in $Y$ of $f^{-1}(p)$ [3, Definition 2].

Theorem 3.5. Let $X$ be a hypotopological space, let $\mu_{X}: X^{\prime} \rightarrow X$ be the canonical presentation of $X$, and let $c l$ and $c l^{\prime}$ represent topological closure in $X$ and $X^{\prime}$ respectively. Then the following are equivalent.

(i) All subspaces of $X$ are strong.

(ii) $\mu_{Y}$ is a pseudo-open map of topological spaces.

(iii) For any subset $T$ of $X, \operatorname{cl}(T)=\mu_{X}\left(\mathrm{cl}^{\prime}\left(\mu_{X}^{-1}(T)\right)\right)$.

(iv) For any subset $T$ of $X, \operatorname{cl}(T)=\bigcup_{C} \operatorname{cl}(C \cap T)$, where $C$ ranges over $\mathscr{K}(X)$.

Proof. By Lemma 3.2, (i) is equivalent to the assertion that $\mu_{x}$ is "hereditarily a quotient map" in the sense of Arhangel'skii [3], and hence is equivalent to (ii) by [3, Th. 1]. (iii) is a rephrasing of the definition of pseudo-openness in terms of closed sets. The equivalence of (iii) and (iv) is immediate from the fact that closure in a disjoint sum is obtained as the union of closures in summands.

A topological space is a Fréchet space if the closure of any subset $S$ consists of $S$ together with the limits of convergent sequences from $S$.

Corollary 3.6. [13, Proposition 7.2] If $X$ is a sequential space with unique sequential limits, then $X$ is hereditarily sequential if and only if $X$ is a Fréchet space.

A collection of subsets $\left\{C_{\alpha}\right\}$ of a topological space $X$ is called an interior cover of $X$ if the interiors of the $C_{\alpha}$ cover $X[8, \mathrm{p} .285]$. 
THeOREM 3.7. If $X$ is a hypotopological space and $\mathscr{K}(X)$ is an interior cover of $X$ (where interiors are taken with respect to the induced topology), then every subset of $X$ is strong.

Proof. If $\mathscr{K}(X)$ is an interior cover, then the presentation map $\mu_{X}$ is pseudo-open.

Corollary 3.8. Let $X$ be a k-space with the full k-hypotopology. If $X$ is locally compact in the sense that every point has a closed compact neighborhood, then every subset of $X$ is strong.

CoRollary 3.9. Let $P$ be the lattice product of a finite number of chains, with the interval hypotopology. Then every subset of $P$ is strong.

Proof. It is easily seen that if hypotopological spaces $X_{1}, \cdots$, $X_{n}$ have the property that $\mathscr{K}\left(X_{i}\right)$ is an interior cover, then so does $X_{1} \times \cdots \times X_{n}$. The intervals of a chain do form an interior cover, and the product hypotopology on $P$ is the interval hypotopology by Lemma 6.3 below.

REMARK. Alo and Frink [1] have shown that the Birkhoff interval topology is preserved under finite lattice products of chains.

4. Chains in partially ordered sets. The main result of this section is that chains in partially ordered sets are always strong.

If $P$ is a partially ordered set and $x \in P$, by the "rays" $M(x)$ and $J(x)$ we shall mean $M(x)=\{y \in P: y \leqq x\}$ and $J(x)=\{y \in P: y \geqq x\}$. $M(x)$ is a closed subset under the Birkhoff interval topology, and similarly for $J(x)$.

LEMma 4.1. Let $S$ be a maximal chain in a partially ordered set $P$. Then $S$ is closed in $P$ under the Birkhoff interval topology.

Proof. Since all sets $M(x)$ and $J(x)$ are closed, the set $T=$ $\bigcap_{x \in S}(M(x) \cup J(x))$ is closed. But $y \in T$ if and only if $y$ is comparable with every element of $S$. Since $S$ is a maximal chain, $T=S$.

REMARK. The relativization to $S$ of the Birkhoff interval topology on $P$ may be stronger than the intrinsic chain topology on $S$. For example, let $P$ be the reals with 0 replaced by two noncomparable elements $0^{\prime}, 0^{\prime \prime}$. $P$ has two maximal chains, each order-isomorphic to the reals, and yet the infinite open interval $\left(0^{\prime}, \infty\right)=\left(0^{\prime \prime}, \infty\right)=\left[0^{\prime}, \infty\right) \cap$ $\left[0^{\prime \prime}, \infty\right)$ is a relatively closed subset of either chain. 
THEOREM 4.2. Let $P$ be a partially ordered set under the interval hypotopology. Then every chain $S$ of $P$ is a strong subset of $P$.

Proof. Extend $S$ to a maximal chain $T$ of $P$. By Lemma 4.1, $T$ is closed in $P$. Then by Theorems 3.3-(i) and 3.4-(ii), it suffices to show that $S$ is a strong subset of $T$ with respect to the relative hypotopology $\mathscr{K}(T)$ on $T$. The topology $\mathscr{K}(T): \mathscr{K}(T)$ is at least as strong as the intrinsic chain topology on $T[6, \mathrm{p} .241]$, since the intervals and rays of $T$ which are subbasic closed sets for the latter topology are also closed in the former. Therefore $T$ retains the property of a chain that its intervals form an interior cover, even when interiors are taken with respect to $\mathscr{K}(T): \mathscr{K}(T)$. By Theorem 3.7, every subset of $T$, in particular $S$, is strong.

5. Hypotopologies as lattices. Since chains are well-behaved under the interval hypotopology, it is appropriate to examine next the "horizontal" sets, i.e., sets consisting of pairwise noncomparable elements. In general, horizontal sets are not as tractable as chains. For example, if $\boldsymbol{R}$ denotes the ordered set of real numbers, the Birkhoff interval topology in $\boldsymbol{R} \times \boldsymbol{R}$ is the ordinary Euclidean topology, and yet it is easy to find maximal horizontal sets which are not closed. Furthermore, Corollary 5.9 will show that horizontal subsets need not be strong with respect to the interval hypotopology, even for conditionally complete lattices.

Nevertheless, certain horizontal sets in atomic lattices do turn out to be strong (Theorem 5.5), and we shall use this fact to give an embedding of hypotopological spaces into lattices.

Let $X$ be a $T_{1}$ hypotopological space. Then there is an obvious lattice associated with $X$, namely $\mathscr{K}(X)$ itself, ordered by set inclusion. For clarity, we shall denote $\mathscr{K}(X)$ regarded as a lattice by $L(X)$, with lattice operations $\vee, \wedge$. The element of $L(X)$ corresponding to a given $C \in \mathscr{K}(X)$ will be denoted by $C^{*}$. For $p \in X,\{p\}^{*}$ will be abbreviated to $p^{*}$, and $f_{X}: X \rightarrow L(X)$ will be the function given by $f_{X}(p)=p^{*} . L(X)$ is a conditionally complete atomic distributive lattice with universal lower bound $0=\varnothing^{*}$.

If $V$ is any subset of $X$, let $V^{(n)}=\left\{C^{*} \in L(X)\right.$ : card $\left.(C)=n\right\}$ for $n=0,1,2, \cdots$. Thus $X^{(1)}$ is the image of $f_{X}$, i.e., the set of atoms of $L(X) . \quad V^{(n)}$ is certainly horizontal, for all $n$.

The following observation is the reason for our construction of $f_{X}$.

Lemma 5.1. Let $X$ be a $T_{1}$ hypotopological space. Then $f_{X}: X \rightarrow$ $L(X)$ is an embedding with respect to the interval hypotopology on $L(X)$. 
Proof. For $C \in \mathscr{K}(X)$, the set image $f_{X}(C)$ is $X^{(1)} \cap\left[0, C^{*}\right]$. Thus the sets $f_{X}(C)$ constitute a subbase of $f_{X}(X)=X^{(1)}$ in the relativized interval hypotopology $\mathscr{K}\left(X^{(1)}\right)$. (It is clear that a subbase for $\mathscr{K}\left(X^{(1)}\right)$ consists of all sets of the form $X^{(1)} \cap\left[D^{*}, C^{*}\right]$, but for $D^{*} \neq 0$ such a set has at most one element and is equal to $\varnothing=$ $X^{(1)} \cap[0,0]$ or to $X^{(1)} \cap\left[0, p^{*}\right]$ for some $p \in X$.) It follows that for $C \subseteq X, f_{X}(C) \in \mathscr{K}\left(X^{(1)}\right)$ if and only if $C \in \mathscr{K}(X)$. Thus $f_{X}$ is a hypotopological homeomorphism of $X$ and $X^{(1)}$, i.e., an embedding of $X$ into $L(X)$.

Our efforts will now be directed towards showing (Theorem 5.6) that $f_{X}$ is an embedding under the induced topologies as well.

If $V$ is any closed subset of $X$, the relative hypotopology $\mathscr{K}(V)$ is given by $\mathscr{K}(V)=\{C \in \mathscr{K}(X): C \subseteq V\}$; thus $L(V)$ can be regarded as a subset, in fact a lattice ideal, of $L(X)$. If $V$ is a closed bounded subset of $X$, then $L(V)=\left[0, V^{*}\right]$.

Lemma 5.2. For any closed subset $V$ of a $T_{1}$ hypotopological space $X, L(V)$ is closed in $L(X)$ under the Birkhoff interval topology.

Proof. For any interval $\left[C^{*}, D^{*}\right]$ of $L(X),\left[C^{*}, D^{*}\right] \cap L(V)=$ $\left[C^{*},(D \cap V)^{*}\right]$.

Lemma 5.3. If $T$ is any Hausdorff topological space and $p_{1}, \cdots$, $p_{n+1}$ are distinct points of $T$, there exist closed subsets $D_{1}, \cdots, D_{n+1}$ of $T$ such that (a) $p_{i} \notin D_{i}$ for all $i$, and (b) any subset of $T$ of cardinality $k \leqq n$ is contained in $D_{i}$ for some $i$.

Proof. For each $i<j$, let $E_{i j}$ and $E_{i j}$ be disjoint open sets with $p_{i} \in E_{i j}, p_{j} \in E_{j i}$. For each $i$ let $D_{i}$ be the complement in $T$ of $\bigcap_{j \neq i} E_{i j}$. Then (a) holds. For $i \neq j, D_{i} \cup D_{j}=T$. Then if $Q \subseteq T$ and card $(Q) \leqq$ $n$, each element of $Q$ is contained in all except possibly one of the $(n+1)$ sets $D_{i}$. It follows that $Q \subseteq D_{i}$ for some $i$.

In view of the "vertical" nature of intervals used in defining the Birkhoff interval topology, the following fact is striking.

Lemma 5.4. Let $X$ be a Hausdorff hypotopological space. Then for every nonnegative integer $n, X^{(0)} \cup \cdots \cup X^{(n)}$ is closed in the Birkhoff interval topology on $L(X)$.

Proof. Let $S=X^{(0)} \cup \cdots \cup X^{(n)}$. $S$ will be its own closure, i.e., closed, if we can show that for every $C^{*} \in L(X)$ with $C^{*} \notin S$ there exists a closed subset $R$ of $L(X)$ with $S \subseteq R, C^{*} \notin R$. Such a $C$ has more than $n$ elements; let $Q \subseteq C$ have $(n+1)$ elements $p_{1}, \cdots, p_{n+1}$. 
Use Lemma 5.3 to choose corresponding closed sets $D_{1}, \cdots, D_{n+1}$ in $X$. Let $R=\bigcup_{i} L\left(D_{i}\right)$. Then $R$ is closed by Lemma 5.2. By (b) of Lemma 5.3, $X^{(k)} \cong R$ for $k \leqq n$, so that $S \subseteq R$. But by (a), we have (for all i) $p_{i}^{*} \in L\left(D_{i}\right)$, hence $Q^{*} \notin L\left(D_{i}\right)$, hence $C^{*} \in L\left(D_{i}\right)$, hence $C^{*} \notin R$. Thus $R$ has the required properties.

Theorem 5.5. Let $X$ be a Hausdorff hypotopological space. For every nonnegative integer $n, X^{(n)}$ is a strong subset of $L(X)$ with respect to the interval hypotopology on $L(X)$.

Proof. $S=X^{(0)} \cup \cdots \cup X^{(n)}$ is closed in $L(X)$ by Lemma 5.4, hence is strong by Theorem 3.3 (i). But $X^{(0)} \cup \cdots \cup X^{(n-1)}$ is also closed in $L(X)$, hence in $S$, and its complement $X^{(n)}$ in $S$ is therefore open in $S$. By Theorem 3.3 (ii), $X^{(n)}$ is strong in $S$, and by Theorem 3.4 (ii), $X^{(n)}$ is strong in $L(X)$.

We shall be interested in Theorem 5.5 particularly for $n=1$ and 2.

THEOREM 5.6. Let $X$ be a Hausdorff hypotopological space. Then $f: X \rightarrow L(X)$ is simultaneously (1) a hypotopological embedding with respect to the interval hypotopology on $L(X)$; and (2) a topological embedding with respect to the induced topology on $X$ and the Birkhoff interval topology on $L(X)$.

Proof. Lemma 5.1 shows that $f_{X}$ is a hypotopological embedding. Hence $f_{X}$ is a topological homeomorphism of the induced topologies of $X$ and of $f_{X}(X)=X^{(1)}$. By Theorem 5.5 for $n=1$, the induced topology on $X^{(1)}$ is the relativized Birkhoff interval topology.

Corollary 5.7. Any Hausdorff sequential topological space $X$ can be obtained by relativization from a conditionally complete lattice with 0 under its Birkhoff interval topology, so that closed bounded subsets of the lattice intersect $X$ in convergent sequences.

Corollary 5.8. Any Hausdorff k-space $X$ can be obtained by relativization from a conditionally complete lattice with 0 , under its Birkhoff interval topology, so that closed bounded subsets of the lattice intersect $X$ in compact sets.

COROLlary 5.9. There exist conditionally complete distributive atomic lattices in which not every subset is strong with respect to the interval hypotopology; in particular, a horizontal subset may not be strong. 
Proof. Let $X$ be a Hausdorff hypotopological space in which not every subset is strong; for example, let $X$ be a Hausdorff sequential space which has a nonsequential subspace [12, Example 1.8]. Consider $f_{X}(S) \subseteq L(X)$, where $S \subseteq X$ is not strong. By Theorem 5.6, if $f_{X}(S)$ were strong in $L(X)$, then $S$ would be strong in $X$, contrary to assumption.

At this point, it is natural to ask which lattices $M$ have the form $L(X)$ for some hypotopological space $X$. And for which of these is $\mathscr{K}(X)$ a $k$-hypotopology? This latter question has an unexpected answer.

THEOREM 5.10. A lattice $M$ has the form $L(X)$ for some $T_{1}$ hypotopological space $X$ if and only if $M$ is a conditionally complete, atomic, distributive lattice. If so, $\mathscr{K}(X)$ is a k-hypotopology if and only if $X^{(1)} \subseteq L(X)=M$ is closed in the topology of order convergence in $M$.

Proof. If $M$ is a conditionally complete, atomic distributive lattice, let $X$ be the set of atoms of $M$ with hypotopology $\{X \cap[0, m]$ : $m \in M\}$. Then $M \cong L(X)$. The converse is obvious. All elements of $\mathscr{K}(X)$ are compact if and only if every filter base $B$ contained in $\mathscr{K}(X)$ has a nonempty intersection [7, Definition 1 p. 93]. But a filter base can be interpreted in $L(X)$ as a downward-directed net which is never 0 , and which therefore dominates a net of $X^{(1)}$. From the definition of order-convergence [6, p. 244], a net in $X^{(1)}$ therefore can converge to 0 if and only if there exists a $B$ with empty intersection. Since a net of atoms can order-converge only to an atom or to 0 , the second assertion of the theorem is proved.

6. Products of lattices. It is known that the product of two $k$-spaces may not be a $k$-space [10, p. 563] and that the product of two sequential spaces may fail to be sequential [12, Example 1.11]. More generally, let us say that a pair of hypotopological spaces, $Y$ and $Z$, is strongly productive if the topology on $Y \times Z$ induced by the product hypotopology is the product of the induced topologies. The following fact is immediate.

Lemma 6.1. Two hypotopological spaces $Y$ and $Z$ are strongly productive if and only if $\mu_{Y} \times \mu_{Z}$ is a quotient map, where $\mu_{Y}: Y^{\prime} \rightarrow$ $Y$ and $\mu_{Z}: Z^{\prime} \rightarrow Z$ are the canonical presentations of $Y$ and $Z$.

THEOREM 6.2. Let $X$ be a Hausdorff hypotopological space. Then the following conditions are equivalent.

(i) For all Hausdorff topological spaces $Z, X$ and $Z$ are strongly 
productive (where $Z$ is regarded as a hypotopological space).

(ii) $\mu_{X}$ is a bi-quotient $\operatorname{map}$ [20]; i.e., if $\left\{U_{\alpha}\right\}$ is an open cover of $\mu_{X}^{-1}(p)(p \in X)$ then for some finite set of indices $\alpha(1), \cdots, \alpha(n)$, the sets $f\left(U_{\alpha(i)}\right)$ cover a neighborhood of $p$.

(iii) $\mathscr{K}(X)$ is an interior cover of $X$.

Proof. (i) $\Leftrightarrow$ (ii). By Lemma 6.1, (i) is equivalent to the assertion that $\mu_{X} \times \mu_{Z}$ is a quotient map for any Hausdorff topological space $Z$. Theorem 1.3 of [20] states that (ii) is equivalent to the assertion that $\mu_{X} \times i_{Z}$ is a quotient map for any such $Z$, where $i_{Z}$ is the identity map on $Z$. It will therefore be sufficient to prove: $\left(^{*}\right)$ For any topological space $Z, \mu_{x} \times \mu_{Z}$ is a quotient map if and only if $\mu_{X} \times i_{Z}$ is a quotient map. Accordingly, let such a $Z$ be given. Since $Z \in \mathscr{K}(Z), Z$ itself is a disjoint summand of $Z^{\prime}$; let $j: Z \rightarrow Z^{\prime}$ be the corresponding embedding. Note that $\mu_{X} \times \mu_{Z}=\left(\mu_{X} \times i_{Z}\right)$ 。 $\left(i_{X}, \times \mu_{z}\right)$ and $\mu_{X} \times i_{z}=\left(\mu_{X} \times \mu_{Z}\right) \circ\left(i_{X}, \times j\right)$. Since the composition of two maps is a quotient map only if the left-hand factor is a quotient map, the assertion $(*)$ is verified.

(ii) $\Rightarrow$ (iii). Let $p \in X$. The various $C \in \mathscr{K}(X)$, regarded as summands of $X^{\prime}$, together form an open cover of $\mu_{X}^{-1}(p)$. By (ii), there exist $C_{1}, \cdots, C_{n} \in \mathscr{K}(X)$ such that the $\mu_{X}\left(C_{i}\right)$ cover a neighborhood of $p$ (taken with respect to the induced topology on $X$ ). But $\mu_{X}$ on $C_{i}$ is just the embedding of $C_{i}$ in $X$. Thus $C_{0}=C_{1} \cup \cdots \cup C_{n}$ is a neighborhood of $p$, and we have $C_{0} \in \mathscr{K}(X), p \in \operatorname{int}_{X} C_{0}$.

(iii) $\Rightarrow$ (ii). Let $p \in X$, and choose $C_{0} \in \mathscr{K}(X)$ with $p \in$ int $_{X} C_{0}$. If $\left\{U_{\alpha}\right\}$ is an open cover of $\mu_{X}^{-1}(p)$, then for some $\alpha, U_{\alpha}$ contains the singleton set $\mu_{X}^{-1}(p) \cap C_{0}$, where $C_{0}$ is now regarded as a summand of $X^{\prime} . \mu_{X}\left(U_{\alpha}\right)$ contains the open neighborhood $\mu_{X}\left(U_{\alpha} \cap\right.$ int $\left.{ }_{X} C_{0}\right)$ of $p$, hence is itself a neighborhood of $p$.

The proof of Theorem 6.2 is thus complete.

A Hausdorff sequential space $X$ satisfies condition 6.2 (iii) if and only if each $p \in X$ has a compact neighborhood consisting of $p$ and at most a countable number of isolated points. A Hausdorff $k$-space $X$ with a $k$-hypotopology satisfies condition 6.2 (iii) if and only if $X$ is locally compact and the hypotopology is the full $k$-hypotopology. Thus for many hypotopological spaces $X$, including the reals with the sequential hypotopology, there exist hypotopological spaces $Z$ which are actually topological, such that $X$ and $Z$ are not strongly productive.

In the case of lattices $X$ under the interval hypotopology, however, Theorem 6.2 does not say when lattices $Z$ are strongly productive with $X$. (And lattices $X$ are not usually Hausdorff.) It is this type of question which a study of embeddings will help to answer (Theorem 
6.6). Alo and Frink [1, Th. 9, p. 1012] have shown that the Birkhoff interval topology is preserved at least for finite products of chains. For the interval hypotopology in place of the Birkhoff interval topology, there is no problem:

Lemma 6.3. If $P$ and $Q$ are partially ordered sets, then the interval hypotopology on $P \times Q$ is the product of the interval hypotopologies of $P$ and $Q$.

Proof. The intervals of $P \times Q$ are precisely the products of intervals of $P$ with intervals of $Q$.

Lemma 6.4. Let $Y$ and $Z$ be Hausdorff hypotopological spaces. Then $Y^{(1)} \times Z^{(1)}$ is a strong subset of the lattice product $L(Y) \times L(Z)$, with respect to the interval hypotopology on the product.

Proof. Let $X$ be the hypotopological disjoint sum $Y+Z$. Then $L(Y) \times L(Z)$ is lattice-isomorphic to $L(X)$. Under this isomorphism, the subset $X^{(2)}$ of $L(X)$ corresponds to the subset

$$
S=\left(Y^{(0)} \times Z^{(2)}\right) \cup\left(Y^{(1)} \times Z^{(1)}\right) \cup\left(Y^{(2)} \times Z^{(0)}\right)
$$

of $L(Y) \times L(Z)$. Since $X^{(2)}$ is strong in $L(X)$ by Theorem 5.5, $S$ is strong in $L(Y) \times L(Z)$. Now observe that $Y^{(1)} \times Z^{(1)}=S \cap T$, where

$$
T=\left(Y^{(0)} \cup Y^{(1)}\right) \times\left(Z^{(0)} \cup Z^{(1)}\right)
$$

in $L(Y) \times L(Z)$. By Lemma 5.4, $Y^{(0)} \cup Y^{(1)}$ and $Z^{(0)} \cup Z^{(1)}$ are closed in $L(Y)$ and $L(Z)$ respectively. Since the induced topology on the product of hypotopological spaces is always at least as strong as the product of the induced topologies, $T$ is closed in $L(Y) \times L(Z)$ with respect to the product hypotopology, which is the interval hypotopology by Lemma 6.3. Then $Y^{(1)} \times Z^{(1)}=S \cap T$ is relatively closed in the strong set $S$, hence is strong in $L(Y) \times L(Z)$, by Theorems 3.3 (i) and 3.4 (ii).

Lemma 6.5. Let $Y$ and $Z$ be Hausdorff hypotopological spaces. Then $f_{Y} \times f_{Z}: Y \times Z \rightarrow L(Y) \times L(Z)$ is an embedding of hypotopological spaces with respect to the interval hypotopology on $L(Y) \times L(Z)$.

Proof. It is easily verified that the product of two embeddings of hypotopological spaces is again an embedding. Lemma 6.3 shows that $L(Y) \times L(Z)$ can be regarded as having the interval hypotopology.

Theorem 6.6. Let $Y$ and $Z$ be Hausdorff hypotopological spaces. 
Then $f_{Y} \times f_{Z}: Y \times Z \rightarrow L(Y) \times L(Z)$ is a topological embedding simultaneously for

(1) the product of the induced topologies on $Y$ and $Z$, and the product of the Birkhoff interval topologies on $L(Y)$ and $L(Z)$; and

(2) the topology on $Y \times Z$ induced by the product hypotopology, and the Birkhoff interval topology on $L(Y) \times L(Z)$.

Proof. $f_{Y}$ and $f_{Z}$ are topological embeddings (Theorem 5.6), and a product of topological embeddings is again an embedding. Thus (1) holds. Lemma 6.5 shows that $f_{Y} \times f_{Z}$ is a hypotopological embedding, and since its image $Y^{(1)} \times Z^{(1)}$ is strong in $L(Y) \times L(Z)$ (Lemma 6.4), $f_{Y} \times f_{Z}$ carries the induced topology of $Y \times Z$ to the relativized Birkhoff interval topology on $Y^{(1)} \times Z^{(1)}$. Thus (2) holds.

CoROLLARY 6.7. If $Y$ and $Z$ are Hausdorff hypotopological spaces which are not strongly productive, then $L(Y)$ and $L(Z)$ are not strongly productive for the interval hypotopology, i.e., the Birkhoff interval topology on $L(Y) \times L(Z)$ is not the topological product of the Birkhoff interval topologies of $L(Y)$ and $L(Z)$.

COROLlaRY 6.8. There exists a conditionally complete, distributive, atomic lattice $M$ such that the Birkhoff interval topology on $M \times M$ is not the product of the Birkhoff interval topology on $M$ with itself.

Proof. Let $M=L(X)$, where $X$ is a Hausdorff $k$-space for which $X \times X$ is not a $k$-space, and where $X$ is given the full $k$-hypotopology. Then Corollary 6.7 applies. (For example, let $X$ be the reals under the half-open-interval topology $[17, \mathrm{p} .59]$.)

It might be conjectured that if one of a pair of lattices is complete, then the pair is strongly productive. For, a complete lattice is compact in the Birkhoff interval topology [5, Th. 3], and compact spaces are well-behaved under products in the case of Hausdorff spaces [19, Th. 3.1]. The following fact provides a wide class of counterexamples to such a conjecture.

COROLlaRY 6.9. Let $X$ be a Hausdorff hypotopological space for which $\mathscr{K}(X)$ is not an interior cover of $X$. Then there exists a complete lattice $M$ such that the Birkhoff interval topology on $L(X) \times M$ is not the topological product of the Birkhoff interval topologies on $L(X)$ and $M$.

Proof. By Theorem 6.2 there exists a hypotopological space $Z$ 
which is actually topological, such that $X$ and $Z$ are not a strongly productive pair. Let $M=L(Z)$ and use Corollary 6.7. $M$ is a complete lattice.

\section{REFERENCES}

1. R. Alo and O. Frink, Topologies of lattice products, Canad. J. Math 18 (1966), 1004-1014.

2. Topologies of chains, Math. Ann. 171 (1967), 239-246.

3. A. Arhangel'skii, Some types of factor mappings, and the relations between classes of topological spaces, Soviet Math. Dokl. 4 (1963), 1726-1729.

4. K. A. Baker, Presentations of topological spaces (to appear)

5. G. Birkhoff, A new interval topology for dually directed sets, Revista Mat. y Fis. Teorica Tucuman 14 (1962), 325-331.

6. L Lattice theory, 3rd. ed., Am. Math. Soc. Colloq. Publ. 25, Providence,

R. I., 1967.

7. N. Bourbaki, Eléments de Mathématique, III, Topologie Generale, 4th. ed., Chap. 1 and 2, Hermann, Paris, 1965.

8. E. Cech, Topological spaces, rev. ed., Interscience, New York, 1966.

9. D. E. Cohen, Spaces with weak topology, Quart. J. Math., Oxford Ser. (2) 5 (1954), 77-80.

10. Ch. H. Dowker, Topology of metric complexes, Amer. J. Math. 74 (1952), 555-577.

11. R. M. Dudley, On sequential convergence, Trans. Amer. Math. Soc. 112 (1964), 487-507.

12. S. P. Franklin, Spaces in which sequences suffice, Fund. Math. 57 (1965), 107-115.

13. __ Spaces in which sequences suffice II, Fund. Math. 61 (1967), 51-56.

14. P. Freyd, Abelian categories, Harper and Row, New York, 1964.

15. O. Frink, Topology in lattices, Trans. Amer. Math. Soc. 51 (1942), 568-582.

16. Sz.-Ts. Hu, Introduction to general topology, Holden-Day, San Francisco, 1966.

17. J. L. Kelley, General topology, van Nostrand, New York, 1955.

18. E. Michael, Locally multiplicatively-convex topological algebras, Mem. Amer. Math.

Soc. \#11, (1952).

19. Local compactness and Cartesian products of quotient maps and k-spaces (to appear)

20. — Bi-quotient maps and Cartesian products of quotient maps (to appear)

21. J. H. C. Whitehead, Combinatorial homotopy I, Bull. Amer. Math. Soc. 55 (1949), 213-245.

Received January 17, 1968.

The California Institute of Technology

AND

University of CAlifornia, Los ANGELES 


\section{PACIFIC JOURNAL OF MATHEMATICS}

\section{EDITORS}

H. ROYDEN

Stanford University

Stanford, California

\section{R. $R$ PHELPS}

University of Washington

Seattle, Washington 98105

\section{J. DugunduI}

Department of Mathematics

University of Southern California

Los Angeles, California 90007

RICHARD ARENS

University of California

Los Angeles, California 90024

\section{ASSOCIATE EDITORS}

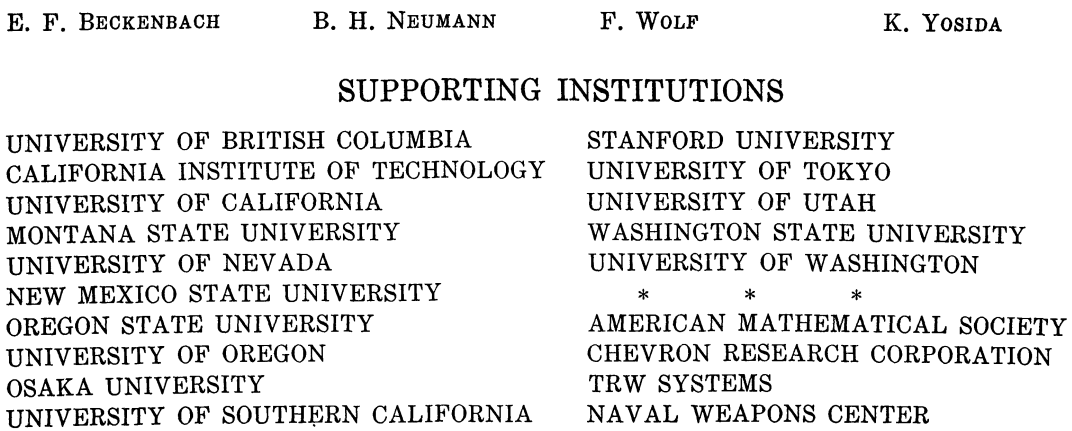

The Supporting Institutions listed above contribute to the cost of publication of this Journal, but they are not owners or publishers and have no responsibility for its content or policies.

Mathematical papers intended for publication in the Pacific Journal of Mathematics should be in typed form or offset-reproduced, double spaced with large margins. Underline Greek letters in red, German in green, and script in blue. The first paragraph or two must be capable of being used separately as a synopsis of the entire paper. It should not contain references to the bibliography. Manuscripts, in duplicate if possible, may be sent to any one of the four editors. Please classify according to the scheme of Math. Rev. 36, 1539-1546. All other communications to the editors should be addressed to the managing editor, Richard Arens, University of California, Los Angeles, California, 90024.

50 reprints are provided free for each article; additional copies may be obtained at cost in multiples of 50 .

The Pacific Journal of Mathematics is published monthly. Effective with Volume 16 the price per volume (3 numbers) is $\$ 8.00$; single issues, $\$ 3.00$. Special price for current issues to individual faculty members of supporting institutions and to individual members of the American Mathematical Society: $\$ 4.00$ per volume; single issues $\$ 1.50$. Back numbers are available.

Subscriptions, orders for back numbers, and changes of address should be sent to Pacific Journal of Mathematics, 103 Highland Boulevard, Berkeley, California, 94708.

PUBLISHED BY PACIFIC JOURNAL OF MATHEMATICS, A NON-PROFIT CORPORATION

Printed at Kokusai Bunken Insatsusha (International Academic Printing Co., Ltd.), 7-17, Fujimi 2-chome, Chiyoda-ku, Tokyo, Japan. 


\section{Pacific Journal of Mathematics}

Vol. 28, No. $2 \quad$ April, 1969

Richard Arens and Donald George Babbitt, The geometry of relativistic

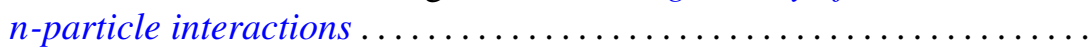

Kirby Alan Baker, Hypotopological spaces and their embeddings in lattices

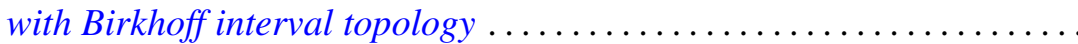

J. Lennart (John) Berggren, Finite groups in which every element is conjugate to its inverse ........................... 289

Beverly L. Brechner, Homeomorphism groups of dendrons . . . . . . . . . . . 295

Robert Ray Colby and Edgar Andrews Rutter, QF - 3 rings with zero singular ideal ................................. 303

Stephen Daniel Comer, Classes without the amalgamation property....... 309

Stephen D. Fisher, Bounded approximation by rational functions ......... 319

Robert Gaines, Continuous dependence for two-point boundary value problems..................................... 327

Bernard Russel Gelbaum, Banach algebra bundles ............... 337

Moses Glasner and Richard Emanuel Katz, Function-theoretic degeneracy criteria for Riemannian manifolds ...................... 351

Fletcher Gross, Fixed-point-free operator groups of order $8 \ldots \ldots \ldots \ldots 357$

Sav Roman Harasymiv, On approximation by dilations of distributions . . . . 363

Cheong Seng Hoo, Nilpotency class of a map and Stasheff's criterion ... . . 375

Richard Emanuel Katz, A note on extremal length and modutus.......... 381

H. L. Krall and I. M. Sheffer, Difference equations for some orthogonal polynomials ................................

Yu-Lee Lee, On the construction of lower radical properties ........... 393

Robert Phillips, Liouville's theorem........................... 397

Yum-Tong Siu, Analytic sheaf cohomology groups of dimension $n$ of

n-dimensional noncompact complex manifolds ..... . .

Michael Samuel Skaff, Vector valued Orlicz spaces. II...

James DeWitt Stein, Homomorphisms of $B^{*}$-algebras .... . .

Mark Lawrence Teply, Torsionfree injective modules .... . . .

Richard R. Tucker, The $\delta^{2}$-process and related topics. II .

David William Walkup and Roger Jean-Baptiste Robert Wets, Lifting

projections of convex polyhedra...

Thomas Paul Whaley, Large sublattices of a lattice. 